\title{
Mining and Harnessing Natural Variation: A Little MAGIC
}

\author{
Gurmukh S. Johal,^ Peter Balint-Kurti, and Clifford F. Weil
}

\begin{abstract}
The success of a breeding program depends on having adequate diversity in the germplasm. However, as advanced breeding stocks and materials are generated, one casualty is the diversity itself. As a result, breeding programs in many crop species have reached a point of diminishing returns and it is feared that unless new diversity is infused into the breeding germplasm, we face catastrophic reductions in crop productivity if the climate turns adverse. Although some scientists favor transgenic approaches, a "back to nature" approach to genetic diversity may prove faster and more effective. Wild and exotic relatives of crop plants hold a wealth of alleles that, if we can find them, can help break yield barriers and enhance tolerance to stresses. Many approaches, based largely on quantitative trait loci genetics, have been proposed and used for this purpose, but most are either highly laborious or discover relevant variation inefficiently. Here, we propose a gene-centered approach, dubbed MAGIC (mutant-assisted gene identification and characterization), that uses Mendelian mutants or other genetic variants in a trait of interest as reporters to identify novel genes and variants for that trait. MAGIC is similar to enhancer-suppressor screens, but rather than relying on variation created in the laboratory, it reveals variation created and refined by nature over millions of years of evolution. This approach could be an effective tool for exploring novel variation and a valuable means to harness natural diversity and define genetic networks.
\end{abstract}

G.S. Johal, Dep. of Botany and Plant Pathology, Purdue Univ., West Lafayette, IN 47907; P. Balint-Kurti, USDA-ARS, Dep. of Plant Pathology, North Carolina State Univ., Raleigh, NC 27695; C.F. Weil, Dep. of Agronomy, Purdue Univ., West Lafayette, IN 47907. Received 14 Mar. 2008. *Corresponding author (gjohal@purdue.edu).

Abbreviations: $\mathrm{CGV}$, cryptic genetic variation; $\mathrm{HR}$, hypersensitive response; MAGIC, mutant-assisted gene identification and characterization; NAM, nested association mapping; QTL, quantitative trait locus; RIL, recombinant inbred line; WT, wild-type.

Lthough Both domestication and modern breeding have
led to present-day crops that are far superior to their wild counterparts, in many cases they also represent a narrowing germplasm pool (Lee, 1998; Tanksley and McCouch, 1997). It is estimated that for most crop species, less than 5\% of the biodiversity known to exist is being utilized in agriculture, particularly in the case of self-pollinated crops (Tanksley and McCouch, 1997). Much of the diversity present in living systems is probably adaptive, and it is feared that our cultivated germplasm is ill equipped to withstand predicted, catastrophic changes in the environment due to global warming. Furthermore, existing germplasm may not have the potential to meet the food needs of an ever-increasing human population, estimated to swell to 9 billion by 2050 (Green et al., 2005). In some respects, narrowing the gene pool has been precisely the goal of breeding: building specific combinations of genes and alleles that are of superior productivity and quality while discarding combinations that are less so. More recently, however, we have learned, or at least been reminded, to look to wild relatives of crop species for useful alleles (e.g., conditioning disease or stress resistance) and to utilize the wealth

\footnotetext{
Published in Crop Sci. 48:2066-2073 (2008).

doi: 10.2135/cropsci2008.03.0150

(C) Crop Science Society of America

677 S. Segoe Rd., Madison, WI 53711 USA

Freely available online through the author-supported open access option.

All rights reserved. No part of this periodical may be reproduced or transmitted in any form or by any means, electronic or mechanical, including photocopying, recording, or any information storage and retrieval system, without permission in writing from the publisher. Permission for printing and for reprinting the material contained herein has been obtained by the publisher.
} 
of natural variation that is accessible (Lee et al., 2002; McCouch, 2004). An additional consideration is the need to stay vigilant of gene-gene interactions, which are often overlooked in complex trait studies (Carlborg and Haley, 2004).

Breeders have not made as much use of the materials in germplasm collection centers (gene banks) as had been anticipated when they were created, largely for want of techniques to discover and then utilize useful variation from them (Hoisington et al., 1999; Tanksley and McCouch, 1997). This reluctance may be, in part, because breeders generally have to contend with upward of 30,000 genes in most crop genomes in their efforts to produce the best-performing genotypes. Alleles at many of these loci have to be either favorable, or at least neutral, for optimal plant performance, a situation typically requiring millennia to achieve for most crops. Replacing one allele at a time with a superior, "alien" allele is done by introgression breeding without having to distort the rest of the collection, easiest if the alien allele causes an easily selectable phenotype (Frisch et al., 1999; Peleman and van der Voort, 2003). But if we think of the genome as a deck of cards, breeders are understandably much less comfortable reshuffling the entire deck of their carefully developed germplasm with that of a wild plant. This is particularly true when a trait of interest is relatively complex and controlled by genes that at least singly, lack an easily observed phenotype.

Advances in quantitative genetics and genomic technologies, and the realization that transgenic technology may not boost plant yields and sustainability as quickly as hoped, have sparked a renewed interest in understanding and using natural variation for crop improvement. A new "agrobiodiversity" discipline has emerged, a synthesis of biodiversity research with genetic resource development (Hammer et al., 2003). Natural variation represents a huge and largely untapped resource, subject to selection over millions of years of evolution, with both basic and practical value, as well as the potential to break yield barriers of agricultural plants (Tanksley and McCouch, 1997; Zamir, 2001). In addition, naturally occurring variation can be harnessed as a gene discovery tool, characterizing interactions between a defined mutation and natural alleles in other genes from various genetic backgrounds. This is similar to the notion of cryptic genetic variation (CGV) that has been described primarily for a few morphological mutations in Drosophila and stickleback fish (Gasterosteus aculeatus) (Gibson and Dworkin, 2004; Shapiro et al., 2004) but also in maize (Zea mays L.) $\times$ teosinte [Zea luxurians (Durieu \& Asch.) Bird] crosses (Lauter and Doebley, 2002). This variation only becomes apparent when it is combined with a mutation of interest. Note that this is not the same hidden variation thought to lie within every organism that is buffered from expression by genes like HSP90 (Queitsch et al., 2002; Sangster et al., 2008). The first reports of CGV arose largely through noticing that Drosophila mutations of interest appeared either suppressed or enhanced when crossed into different inbred backgrounds that otherwise showed no noticeable mutant phenotype for the trait (Gibson and Dworkin, 2004). Lauter and Doebley (2002) then reasoned that this type of variation could play an important role as the domestication of a crop from a wild species plays out over time, and they used quantitative trait locus (QTL) analysis to identify over 20 modifiers of plant architecture that showed up in crosses of maize with its progenitor, teosinte, but that showed no variation among teosintes. The evolutionary importance of such cryptic variation is also of interest (Le Rouzic and Carlborg, 2008).

Ironically, CGV is often observed and deliberately discarded as the "background effects" that can confound the early analysis of a mutation. However, recent detailed and publicly available characterization of diverse germplasm for species like maize (Flint-Garcia et al., 2005; Whitt and Buckler, 2003; Yu et al., 2008) and Arabidopsis (AlonsoBlanco et al., 2005; de Meaux and Koornneef, 2008; Keurentjes et al., 2008; Koornneef et al., 2004) suggest that we should reexamine these background effects. A methodical and large-scale approach to understanding what genes underlie these effects is now possible with QTL approaches (Doerge, 2002; Koornneef and Stam, 2001; Koornneef et al., 2004; McMullen, 2003; Salvi and Tuberosa, 2005; Tanksley and Nelson, 1996) and can reveal the genetic and functional interactions that are one of the grand challenges of plant biology in the post-genome-sequencing era. However, we also need to reexamine genetic background effects and methods for identifying such background effects have been developed (Jannink, 2007a,b; Jannink and Jansen, 2001; Li et al., 2005; Yang et al., 2008).

\section{THE IMPORTANCE OF PRECISION PHENOTYPING}

To make the best use of QTL approaches to identifying these genetic networks, a key component of this strategy is measuring small changes in a wide range of phenotypes of interest accurately, as quantitatively as possible and across several hundred plants. Types of data include morphological and biochemical data, as well as transcriptome profiling, proteomics, metabolic profiling, and ionomics. This goal remains a formidable challenge, however, especially for traits of agricultural importance, and phenotyping techniques and platforms have become perhaps the most important, limiting factors in our ability to make use of natural variation for crop improvement. Recent discussions have focused on applying a phenomics approach to generate and integrate phenotypic data using a number of technologies, old and new, invasive and noninvasive (Edwards and Batley, 2004; Montes et al., 2007). 
These large-scale approaches will probably require coordination at national and international levels to be fully effective, which invariably solves some problems while creating others. Still, high-throughput, precision phenotyping is likely to have a revolutionary impact, both on QTL analysis and on our ability to detect and make use of natural variation.

For those species for which establishing and characterizing diversity collections is still in progress, it is worth noting here that the "cryptic" part of cryptic genetic variation suggests that phenotype will not necessarily be a good predictor in choosing diverse germplasm, and can even be misleading. For example, a given accession may have two or more genes with opposite effects on the trait of interest, and their combined action could keep the favorable gene masked (Hake and Rocheford, 2004; Mitchell-Olds and Schmitt, 2006). In addition, a given exotic line with a null allele for one step in a pathway leading to a trait of interest may also harbor potentially superior alleles of other genes in that pathway or that regulate the pathway (Fig. 1; Bernacchi et al., 1998). Accordingly, useful diversity collections can instead be established and expanded by combining geographic distribution and molecular marker criteria. Along similar lines, over a decade ago Tanksley and McCouch (1997) stressed that "the paradigm [in plant breeding] needs to shift away from selecting potential parents on the basis of phenotype to evaluating them directly for the presence of useful genes."

\section{MUTANT-ASSISTED GENE IDENTIFICATION AND CHARACTERIZATION (MAGIC)}

Regardless of the phenotypic platform, once a phenotypic assay is established, genetic modifiers can be identified. Conceptually, broad, systematic mining of CGV for enhancers and suppressors of genes of interest is similar to the more familiar enhancer-suppressor screens performed using mutagenesis (Fig. 2; Page and Grossniklaus, 2002). The difference lies in the source of the genetic variation providing the modifiers and the use of the extensive mapping in diversity collections and derivatives of them to identify the interacting genes. In addition, the modifying alleles discovered using natural variation have the advantage that they have been filtered by natural selection, likely minimizing any deleterious side effects (while enhancing enormously the chances of identifying genetic variants that can have adaptive importance in agriculture).

It is well known that genetic background influences the penetrance (whether or not a given phenotype is detected) and expressivity (the strength of a given phenotype) of mutations. As a result, after first identifying a new mutation of interest, one introgresses the mutation into an inbred background to clarify the inheritance pattern of the mutation. Typically, a mutation is crossed into several inbreds to find the background that yields the most consistent and pronounced phenotype (Freeling and Fowler, 1993). As mentioned above, the variation revealed when assessing mutations in different genetic backgrounds is often regarded as a problem for comparing effects of different genes influencing the same trait. Putting all such mutations in the same genetic background is thought to be important for comparing and contrasting phenotypes.

From the standpoint of CGV, however, the observed background effects for a mutation in these different inbreds is a valuable resource for discovering interacting QTLs and the genes underlying those QTLs. Although lines known to carry a mutation are often remutagenized to find interacting factors (Fig. 2), natural variation often provides similar alleles as genetic background effects. Many studies have now revealed that these genetic modifiers, as loci that have a quantitative or qualitative impact on the trait of interest, should be considered QTLs and analyzed as such (Abiola et al., 2003; Dworkin, 2005; Nadeau, 2005).

We have coined the name MAGIC (mutant-assisted gene identification and characterization) for a broad and deliberate mutant enhancer-suppressor analysis based on natural variation. MAGIC is capable of aiding in the identification and characterization of genetic modifiers and QTLs in highly diverse germplasm and can serve as an effective and efficient tool for gene discovery by assessing and surveying useful variation for a given trait in diverse germplasm. This approach can be used with both dominant and recessive mutations and can detect all classes of modifiers/QTLs, dominant and recessive, major and minor, additive and nonadditive, epistatic and hypostatic (Phillips, 1998). As well as being a tool for uncovering previously undetectable QTLs, MAGIC can simplify the scoring changes in traits that may otherwise be difficult or labor intensive to score (see example of Rp1-D21 below), thereby alleviating some of the problems associated with generating good phenotypic data.

The protocol for MAGIC will differ slightly depending on whether the initial, "reporter" mutation is recessive or dominant or partially dominant. If the mutation is recessive, it is crossed with diverse germplasm lines, and the resulting $\mathrm{F}_{1}$ hybrids are self-pollinated to generate $F_{2}$ populations. About 50 plants from each of these $\mathrm{F}_{2}$ populations are then phenotyped for transgressive changes in the expression of the reporter mutant (Fig. 3 ). The diverse parent of any $F_{2}$ populations in which the phenotype of the mutant has been either enhanced or suppressed is thus identified as having genes/QTLs relevant to the trait of interest, with the initial mutation serving as the readout for these QTLs/modifiers. With $50 \mathrm{~F}_{2}$ progeny, enough phenotypic variation should be present to detect enhanced and/or suppressed classes of transgressive changes in the mutant phenotype. 
Either of two routes can be followed at this point depending on what resources have been developed. For most systems, more progeny from any appropriate $\mathrm{F}_{2}$ population can be planted to identify at least 100 plants with the mutant phenotype. These mutants can then be phenotyped using a quantitative measure of the overall severity of the mutant phenotype, and then genotyped. The resulting data from these mutant progeny can be assessed for QTLs to identify and localize relevant loci. Even though this approach requires extensive genotyping, it is only on lines that are already shown to segregate for modifiers of interest and should produce data sufficient to narrow the position of the modifier to a manageable region. Extreme $\mathrm{F}_{2}$ mutant segregants with enhanced or suppressed phenotypes are also of interest because they have pyramided most modifier/QTL alleles that impact the trait of interest either negatively or positively. In fact, this idea has been used to enhance the performance of two maize mutants, shrunken2 (sh2) and opaque2 (o2), respectively, for commercial production of supersweet corn and high-quality protein maize (Prasanna et al., 2001; Tracy, 1997).

However, for some systems all this genotyping has already been done and made available, notably maize

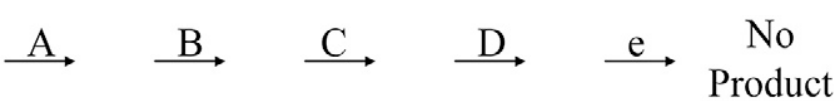

Figure 1. A hypothetical biosynthetic pathway controlled by five genes. The gene (e) controlling the last step is defective, causing little or no product being synthesized.

(http://www.panzea.org) and Arabidopsis (http://walnut.usc.edu/2010); large public projects have developed recombinant inbred lines from the crosses of the diverse germplasm lines and the sequenced reference genome: $\mathrm{B} 73$ in the case of maize, creating the nested association mapping panel (Yu et al., 2008), and Col-O in the case of Arabidopsis (http://walnut.usc.edu/2010). Once it becomes clear which diverse parents contain modifiers of the reporter mutation, the reporter mutant is crossed to the set of recombinant inbred lines derived from that diverse parent, and then selfed. These $\mathrm{F}_{2}$ lines are then screened for the same transgressive effects originally observed when the reporter was crossed to the diverse parent of those recombinant inbred lines (RILs). Extensive genotyping of the RILs (targets of tens or even hundreds of thousands of markers) is already done and publicly available, such that the segments of the diverse and the reference genomes present

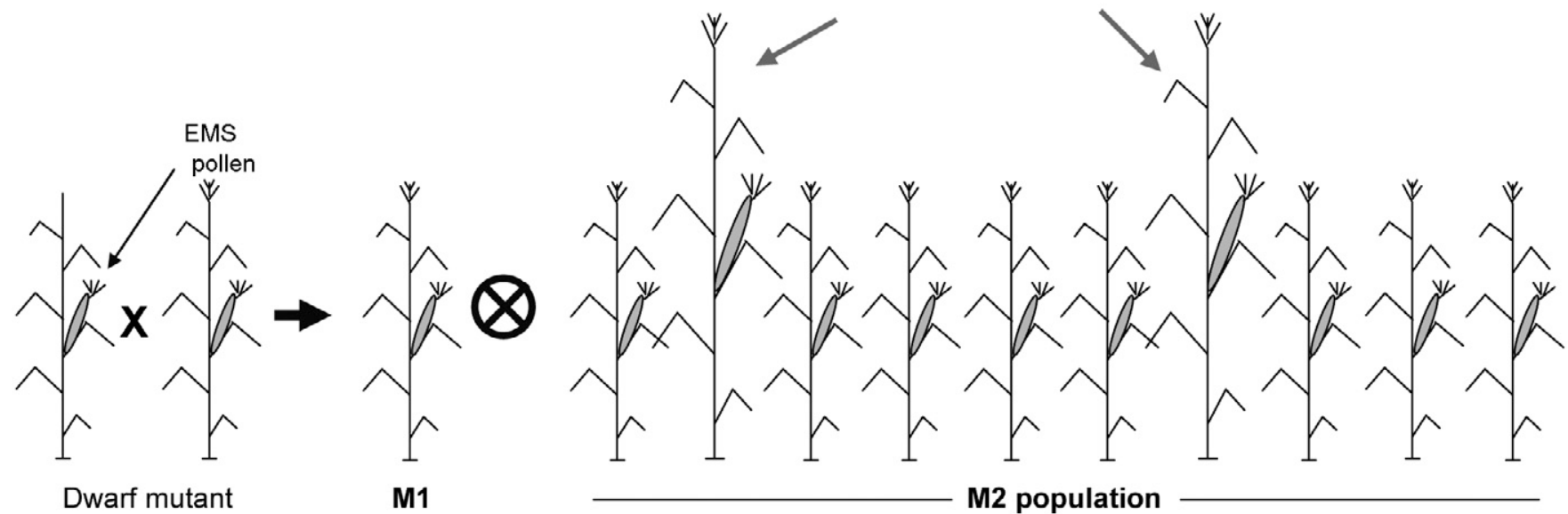

Figure 2. Second site suppression mutagenesis approach. M2 plants with arrows represent revertants in which the effect of the mutant phenotype has reversed. EMS, ethyl methane sulfonate; M1, the first generation following mutagenesis; M2, progeny of M1 individuals derived by self-pollination.

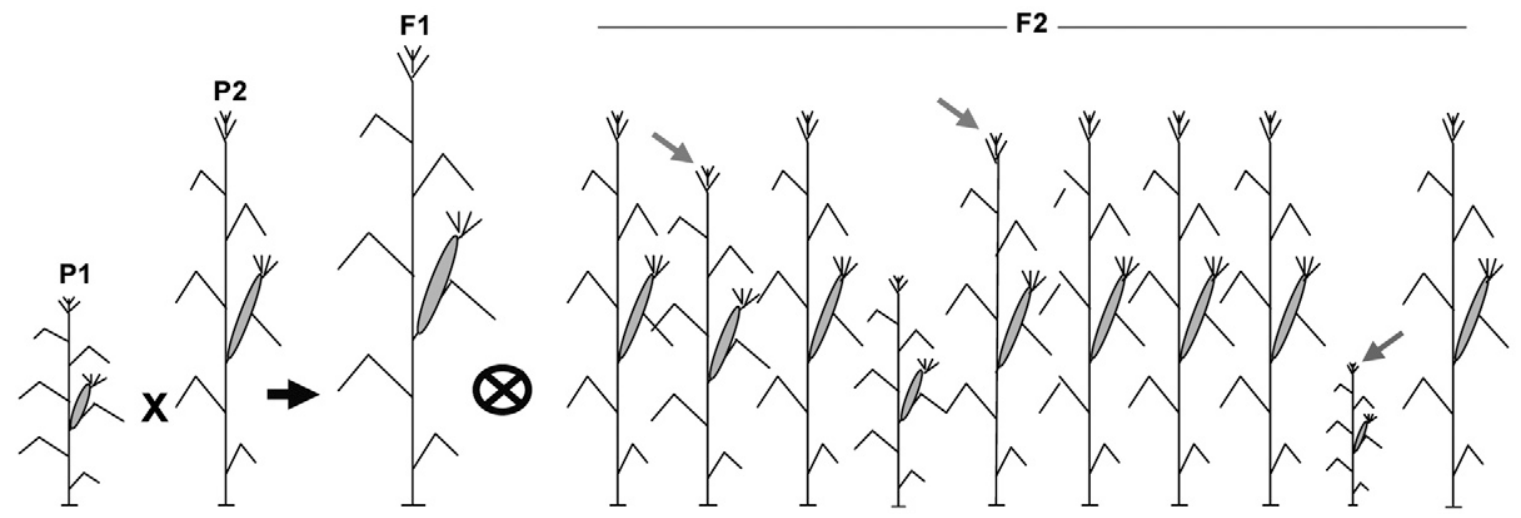

Figure 3. Mutant-assisted gene identification and characterization (MAGIC) using a recessive reporter mutation (P1). Arrows facing right point to F2 mutant segregants with a suppressed phenotype. The segregant with a left-facing arrow has an enhanced mutant phenotype. 
in each RIL are very well understood. Common segments present in those RILs that carry modifiers thus define narrow candidate regions that carry those modifiers. As a result, no additional genotyping is required at all.

If the reporter mutation is dominant or partially dominant, then phenotypic evaluations to identify relevant diversity lines with QTLs of interest can begin in the $\mathrm{F}_{1}$ generation. Following crosses with the panel of diverse germplasm, the $\mathrm{F}_{1}$ progeny (as few as 10 plants for each) can be assessed for changes in the mutant phenotype. It is good idea to use the reporter mutant as a heterozygote because, in addition to showing that the cross to the diverse lines is genuine, segregation of the mutant phenotype can also help reveal whether any suppression of the mutant phenotype is truly due to trait-specific QTLs or simply the result of heterotic vigor of the cross. If the diverse inbred line contains a dominant suppressor or enhancer of the reporter mutation, then the $\mathrm{F}_{1}$ progeny will have either a uniformly suppressed or enhanced phenotype, respectively (Fig. 4). Moreover, comparison of the mutant versus wild-type (WT) siblings (e.g., height ratio) can provide additional phenotypic measurements that could prove useful in revealing and mapping QTLs effectively. As mentioned above, the availability of sets of well-characterized RILs developed from the panel of diversity lines can then narrow the location of any modifiers quickly with little or no need for additional genotyping. If the modifier is recessive, an extra generation will be required, best done as a backcross of the $\mathrm{F}_{1}$ to the diverse parent (or the $\mathrm{F}_{1} \mathrm{WT} \times \mathrm{F}_{1}$ mutant sib to generate an $\mathrm{F}_{2}$ ) rather than selfing the $F_{1}$ to ensure that any changes result from the modifier and not from changes in dosage of the reporter mutation. If resources like those in maize and Arabidopsis are not available (which is the case for most crop plants), then to locate genes/QTLs revealed in diverse germplasm, one needs to go to $\mathrm{BC}_{1}$ or $\mathrm{F}_{2}$ populations.

\section{An Example}

Maize disease lesion mimic (les) mutants provide a particularly good example of how the MAGIC approach can be applied. These mutants show spontaneous cell death resembling what is caused by pathogen attack, but they do so in the absence of the pathogen (Johal, 2007; Johal et al., 1995; Neuffer and Calvert, 1975). Some maize les mutants can have a lethal phenotype in one genetic background and yet a nearly undetectable, benign phenotype in another (Johal, 2007; Neuffer et al., 1983). Their easily discerned, visible phenotype and their sensitivity to background effects make les mutations excellent reporters of CGV. For example, an $\mathrm{F}_{2}$ population was developed between les23, a recessive allele characterized by formation of yellowish-brown, cell death patches on leaves, and
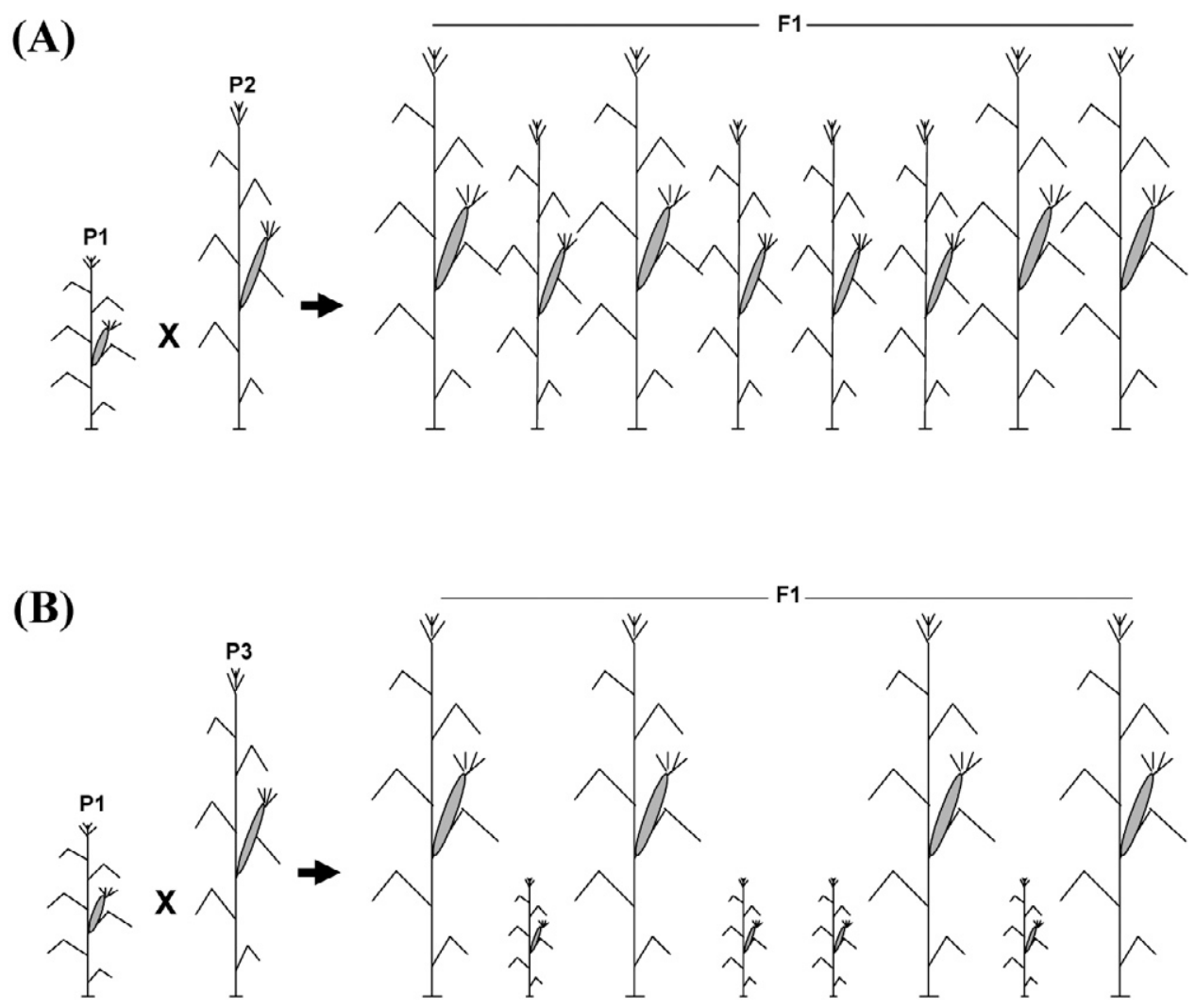

Figure 4. Conducting mutant-assisted gene identification and characterization (MAGIC) with a dominant reporter mutation, used here as a heterozygote in an otherwise pure-breeding line (P1). P2 and P3 are two diverse lines. While P2 has a suppressible effect on the dwarfing phenotype of the mutant (A), P3 has an enhancing effect on this dwarfing mutant (B). In both (A) and (B), families are segregating 1:1 for mutant and wild-type sibs. 
the inbred Mo20W, known to suppress a number of les mutations (Penning et al., 2004). All les 23 mutants, which constituted about one-fourth of the F2 population, were assessed for phenotypic severity (degree of lesioning on leaves scored using a numbering scale) and genotyped. Subsequent QTL analysis identified a major suppressor of cell death $(\operatorname{Slm} 1)$ that accounted for $\sim 70$ and $\sim 90 \%$ of the phenotypic variation present in this $\mathrm{F}_{2}$ population (Penning et al., 2004). Three minor QTLs were also identified that, when combined with $\operatorname{Sim} 1$, completely suppressed cell death associated with les 23 lesions. In contrast, les 23 mutants that lacked all four QTLs had a phenotype more severe than les 23 in its original background.

Crossing to diverse inbreds with follow-up in RILs developed from those inbreds, MAGIC is also well demonstrated using lesion mimics. A dominant Les mutation, Rp1-D21,in the maize inbred H95, has a necrotic phenotype resulting from constitutive activation of the hypersensitive response (HR) (Sun et al., 2001). This mutant was crossed to two inbreds, B73 and Mo17, that are also the parents of the very well characterized IBM RIL population (Lee et al., 2002). While B73 suppressed the necrotic phenotype significantly, Mo17 enhanced it. Rp1-D21 was then crossed to each of the IBM RILs, and the phenotypic data from the crosses together with the publicly available genotypic data of the IBM RILs were used to identify a major QTL from B73 that significantly suppresses the HR phenotype of Rp1-D21 (Balint-Kurti and Johal, unpublished results). We have since widened our studies to encompass the broader range of diverse maize germplasm, using the core set of 25 maize diversity lines (Liu et al., 2003) that are the founders of the nested association mapping (NAM) population. These crosses reveal much more dramatic enhancement and suppression of Rp1-D21, ranging from plants that are completely necrotic (crossed to the inbreds M37w and NC350) to plants with barely detectable lesions (crossed to the inbreds B97, CML333, Oh43, and Tzi8). Crosses to the relevant RILs of the NAM populations (Yu et al., 2008), with their extensive genotyping data, are in progress and should reveal additional QTLs that interact with Rp1-D21. We are also applying this approach to the recessive, sucrose-accumulating mutant tie-dyed1 (Braun et al., 2006) and have crossed it to the NAM founders as well as the larger set of approximately 260 maize diversity lines (Flint-Garcia et al., 2005; Liu et al., 2003) to identify interacting genes controlling the response to increased sugar content.

\section{CONCLUDING REMARKS}

A key advantage of MAGIC is that it provides a simple genetic trick to quickly survey the diverse and alien germplasm for useful genes or alleles in a directed fashion. In addition, this approach has tremendous potential for helping to define genes and genetic networks underlying any trait quickly, and it underscores the value both in defining diverse inbred germplasm and in developing and characterizing RILs from that germplasm. The genetic resources to do both these things are, at least for many crop and model species, readily available. Once in hand, analysis becomes as straightforward as phenotyping a trait of interest and then taking advantage of a commonly available genotyping dataset. Can a single mutation affecting a trait of interest unveil all the QTLs that might impact the same trait? It is unlikely that MAGIC will reveal entire genetic networks in one experiment; however, using new variants that emerge from a MAGIC screen as the starting material for additional MAGIC screens can be a rapid way to fill in gaps in genetic networks and advance taking systems approaches to address a wide array of agricultural questions.

The mutants needed to conduct MAGIC screens can come directly from the large mutant collections available for many crop species. Additional transposon mutagenesis and TILLING projects that are underway for many crop species will serve as an excellent source for more such mutants, especially when they are in uniform, well-characterized backgrounds (Henikoff et al., 2004; Till et al., 2004). Transgenic dominant negative (RNAi) or overexpression mutants will also be particularly valuable starting material, revealing QTLs for the trait of interest in a single generation, just as dominant genetic mutations do (Fig. 3). MAGIC can also be used on cytoplasmic traits by identifying modifiers/QTLs of these traits that are nuclear genes. In fact, restorers of fertility $(\mathrm{R} f)$ in cytoplasmic male sterile maize were identified using essentially this approach (Duvick, 1956).

Certainly, an advantage of MAGIC is that one can capitalize on existing banks of genotyping very quickly. Even for species where such databases are not yet in place, concerted effort can quickly put them in place and make them a community resource. Selection of a panel of diverse germplasm can be based on a combination of geographic distribution and a genomewide scan of a few hundred simple sequence repeat and single nucleotide polymorphism markers. While development of the RILs from this diversity panel crossed to a reference type is a more significant and somewhat longer term challenge, potentially requiring several years, the diversity panel itself can meanwhile serve as a useful venue for QTL mapping. If desired, the numbers of plants that need to be genotyped and phenotyped to reveal QTLs can be limited to those crosses with diversity lines that show the greatest enhancing or suppressing effects. While this is still potentially several hundred plants $X$ several hundred markers, the outcome is highly likely to identify QTLs of significant effect. Pooling the most extreme, transgressive segregants at each end of the spectrum into single, bulked samples and comparing them will also facilitate finding the QTLs of largest 
effect. However, if the idea is to dissect the entire genetic architecture of a trait of interest, every mutant progeny will need to be genotyped and phenotyped carefully. Note also that, for a recessive mutation, after outcrossing to the diverse germplasm and selfing, only plants showing some version of the mutant phenotype (the one-quarter of the total population homozygous for the initial mutation) would need to be genotyped and phenotyped.

In contrast to many QTL analyses, MAGIC starts out with a clearly defined mutation in a well-characterized gene and then identifies QTLs that impact the same trait. The relationship between the starting mutation and interacting factors can be more clearly defined because the effects of the initial mutation are clearly distinguishable from any emerging QTLs. In a typical QTL study, at the outset, one may not yet be aware of what genes are involved in a trait, a range of mutant alleles can all emerge as major QTLs, and understanding which interact with which must then be teased apart. Both approaches are valuable.

The background effects that influence expression of nearly every gene studied to date underscore the idea that, to paraphrase, "no gene is an island," operating alone. While no gene interacts with all other genes in the genome, most genes interact with at least some other genes. Granted, in some cases, one gene accounts for the vast majority of the variation (classically Mendelian genes that appear to show little background effect), but as our ability to phenotype becomes more and more precise, our ability to distinguish these subtle interactors and modulators will improve as well.

\section{Acknowledgments}

We are grateful to Drs. Rebecca Doerge, Mitch Tuinstra, and B.M. Prasanna and two anonymous reviewers for critically reading the manuscript and for providing valuable comments and suggestions.

\section{References}

Abiola, O., J.M. Angel, P. Avner, A.A. Bachmanov, J.K. Belknap, B. Bennett, E.P. Blankenhorn, D.A. Blizard, V. Bolivar, G.A. Brockmann, K.J. Buck, J.F. Bureau, W.L. Casley, E.J. Chesler, J.M. Cheverud, G.A. Churchill, M. Cook, J.C. Crabbe, W.E. Crusio, A. Darvasi, G. de Haan, P. Dermant, R.W. Doerge, R.W. Elliot, C.R. Farber, L. Flaherty, J. Flint, H. Gershenfeld, J.P. Gibson, J. Gu, W. Gu, H. Himmelbauer, R. Hitzemann, H.C. Hsu, K. Hunter, F.F. Iraqi, R.C. Jansen, T.E. Johnson, B.C. Jones, G. Kempermann, F. Lammert, L. Lu, K.F. Manly, D.B. Matthews, J.F. Medrano, M. Mehrabian, G. Mittlemann, B.A. Mock, J.S. Mogil, X. Montagutelli, G. Morahan, J.D. Mountz, H. Nagase, R.S. Nowakowski, B.F. O’Hara, A.V. Osadchuk, B. Paigen, A.A. Palmer, J.L. Peirce, D. Pomp, M. Rosemann, G.D. Rosen, L.C. Schalkwyk, Z. Seltzer, S. Settle, K. Shimomura, S. Shou, J.M. Sikela, L.D. Siracusa, J.L. Spearow, C. Teuscher, D.W. Threadgill, L.A. Toth, A.A. Toye, C. Vadasz, G. Van Zant, E. Wakeland, R.W.
Williams, H.G. Zhang, and F. Zou. 2003. The nature and identification of quantitative trait loci: A community's view. Nat. Rev. Genet. 4:911-916.

Alonso-Blanco, C., B. Mendez-Vigo, and M. Koornneef. 2005. From phenotypic to molecular polymorphisms involved in naturally occurring variation of plant development. Int. J. Dev. Biol. 49:717-732.

Bernacchi, D., T. Beck-Bunn, Y. Eshed, J. Lopez, V. Petiard, J. Uhlig, D. Zamir, and S. Tanksley. 1998. Advanced backcross QTL analysis in tomato: I. Identification of QTLs for traits of agronomic importance from Lycopersicon hirsutum. Theor. Appl. Genet. 97:381-397.

Braun, D.M., Y. Ma, N. Inada, M.G. Muszynski, and R.F. Baker. 2006. tie-dyed1 regulates carbohydrate accumulation in maize leaves. Plant Physiol. 142:1511-1522.

Carlborg, O., and C.S. Haley. 2004. Epistasis: Too often neglected in complex trait studies? Nat. Rev. Genet. 5:618-625.

de Meaux, J., and M. Koornneef. 2008. The cause and consequences of natural variation: The genome era takes off! Curr. Opin. Plant Biol. 11:99-102.

Doerge, R.W. 2002. Mapping and analysis of quantitative trait loci in experimental populations. Nat. Rev. Genet. 3:43-52.

Duvick, D.N. 1956. Allelism and comparative genetics of fertility restoration of cytoplasmically pollen sterile maize. Genetics 41:544-565.

Dworkin, I. 2005. Towards a genetic architecture of cryptic genetic variation and genetic assimilation: The contribution of K.G. Bateman. J. Genet. 84:223-226.

Edwards, D., and J. Batley. 2004. Plant bioinformatics: From genome to phenome. Trends Biotechnol. 22:232-237.

Flint-Garcia, S.A., A.C. Thuillet, J. Yu, G. Pressoir, S.M. Romero, S.E. Mitchell, J. Doebley, S. Kresovich, M.M. Goodman, and E.S. Buckler. 2005. Maize association population: A highresolution platform for quantitative trait locus dissection. Plant J. 44:1054-1064.

Freeling, M., and J. Fowler. 1993. A nine-step way to characterize a morphological mutant. p. 209-211. In M.F.a.V. Walbot (ed.) The maize handbook. Springer, New York.

Frisch, M., M. Bohn, and A.E. Melchinger. 1999. Comparison of selection strategies for marker-assisted backcrossing of a gene. Crop Sci. 39:1295-1301.

Gibson, G., and I. Dworkin. 2004. Uncovering cryptic genetic variation. Nat. Rev. Genet. 5:681-690.

Green, R.E., S.J. Cornell, J.P. Scharlemann, and A. Balmford. 2005. Farming and the fate of wild nature. Science 307:550-555.

Hake, S., and T. Rocheford. 2004. Exploiting quantitative trait loci in gene discovery. Genes Dev. 18:597-601.

Hammer, K., N. Arrowsmith, and T. Gladis. 2003. Agrobiodiversity with emphasis on plant genetic resources. Naturwissenschaften 90:241-250.

Henikoff, S., B.J. Till, and L. Comai. 2004. TILlING: Traditional mutagenesis meets functional genomics. Plant Physiol. 135:630-636.

Hoisington, D., M. Khairallah, T. Reeves, J.M. Ribaut, B. Skovmand, S. Taba, and M. Warburton. 1999. Plant genetic resources: What can they contribute toward increased crop productivity? Proc. Natl. Acad. Sci. USA 96:5937-5943.

Jannink, J.L. 2007a. Identifying quantitative trait locus by genetic background interactions in association studies. Genetics 176:553-561.

Jannink, J.L. 2007b. QTL $\times$ genetic background interaction: Application to predicting progeny value. Euphytica 161:61-69. 
Jannink, J.L., and R. Jansen. 2001. Mapping epistatic quantitative trait loci with one-dimensional genome searches. Genetics 157:445-454.

Johal, G.S. 2007. Disease lesion mimic mutants of maize. Available at http://www.apsnet.org/online/feature/mimics/ (verified 4 Sept. 2008). American Phytopathological Soc., St. Paul, MN.

Johal, G.S., S.H. Hulbert, and S.P. Briggs. 1995. Disease lesion mimics of maize: A model for cell-death in plants. Bioessays 17:685-692.

Keurentjes, J.J., M. Koornneef, and D. Vreugdenhil. 2008. Quantitative genetics in the age of omics. Curr. Opin. Plant Biol. 11:123-128.

Koornneef, M., and P. Stam. 2001. Changing paradigms in plant breeding. Plant Physiol. 125:156-159.

Koornneef, M., C. Alonso-Blanco, and D. Vreugdenhil. 2004. Naturally occurring genetic variation in Arabidopsis thaliana. Annu. Rev. Plant Biol. 55:141-172.

Lauter, N., and J. Doebley. 2002. Genetic variation for phenotypically invariant traits detected in teosinte: Implications for the evolution of novel forms. Genetics 160:333-342.

Lee, M. 1998. Genome projects and gene pools: New germplasm for plant breeding? Proc. Natl. Acad. Sci. USA 95:2001-2004.

Lee, M., N. Sharopova, W.D. Beavis, D. Grant, M. Katt, D. Blair, and A. Hallauer. 2002. Expanding the genetic map of maize with the intermated B73 $\times$ Mo17 (IBM) population. Plant Mol. Biol. 48:453-461.

Le Rouzic, A., and O. Carlborg. 2008. Evolutionary potential of hidden genetic variation. Trends Ecol. Evol. 23:33-37.

Li, R., M.A. Lyons, H. Wittenburg, B. Paigen, and G.A. Churchill. 2005. Combining data from multiple inbred line crosses improves the power and resolution of quantitative trait loci mapping. Genetics 169:1699-1709.

Liu, K., M. Goodman, S. Muse, J.S. Smith, E. Buckler, and J. Doebley. 2003. Genetic structure and diversity among maize inbred lines as inferred from DNA microsatellites. Genetics 165:2117-2128.

McCouch, S. 2004. Diversifying selection in plant breeding. PLoS Biol. 2:e347, doi:10.1371/journal.pbio.0020347.

McMullen, M.D. 2003. Quantitative trait locus analysis as a gene discovery tool. Methods Mol. Biol. 236:141-154.

Mitchell-Olds, T., and J. Schmitt. 2006. Genetic mechanisms and evolutionary significance of natural variation in Arabidopsis. Nature 441:947-952.

Montes, J.M., A.E. Melchinger, and J.C. Reif. 2007. Novel throughput phenotyping platforms in plant genetic studies. Trends Plant Sci. 12:433-436.

Nadeau, J.H. 2005. Listening to genetic background noise. N. Engl. J. Med. 352:1598-1599.

Neuffer, M.G., and O.H. Calvert. 1975. Dominant disease lesion mimics in maize. J. Hered. 66:265-270.

Neuffer, M.G., D.A. Hoisington, V. Walbot, and S.E. Pawar. 1983. The genetic control of disease symptoms. Oxford and IBH, New Delhi, India.
Page, D.R., and U. Grossniklaus. 2002. The art and design of genetic screens: Arabidopsis thaliana. Nat. Rev. Genet. 3:124-136.

Peleman, J.D., and J.R. van der Voort. 2003. Breeding by design. Trends Plant Sci. 8:330-334.

Penning, B.W., G.S. Johal, and M.D. McMullen. 2004. A major suppressor of cell death, slm1, modifies the expression of the maize (Zea mays L.) lesion mimic mutation les23. Genome 47:961-969.

Phillips, P.C. 1998. The language of gene interaction. Genetics 149:1167-1171.

Prasanna, B.M., S.K. Vasal, B. Kassahun, and N.N. Singh. 2001. Quality protein maize. Curr. Sci. 81:1308-1319.

Queitsch, C., T.A. Sangster, and S. Lindquist. 2002. Hsp90 as a capacitor of phenotypic variation. Nature 417:618-624.

Salvi, S., and R. Tuberosa. 2005. To clone or not to clone plant QTLs: Present and future challenges. Trends Plant Sci. 10:297-304.

Sangster, T.A., N. Salathia, H.N. Lee, E. Watanabe, K. Schellenberg, K. Morneau, H. Wang, S. Undurraga, C. Queitsch, and S. Lindquist. 2008. HSP90-buffered genetic variation is common in Arabidopsis thaliana. Proc. Natl. Acad. Sci. USA 105:2969-2974.

Shapiro, M.D., M.E. Marks, C.L. Peichel, B.K. Blackman, K.S. Nereng, B. Jonsson, D. Schluter, and D.M. Kingsley. 2004. Genetic and developmental basis of evolutionary pelvic reduction in threespine sticklebacks. Nature 428:717-723.

Sun, Q., N.C. Collins, M. Ayliffe, S.M. Smith, J. Drake, T. Pryor, and S.H. Hulbert. 2001. Recombination between paralogues at the Rp1 rust resistance locus in maize. Genetics 158:423-438.

Tanksley, S., and J.C. Nelson. 1996. Advanced backcross QTL analysis: A method for the simultaneous discovery and transfer of valuable QTLs from unadapted germplasm into elite breeding lines. Theor. Appl. Genet. 92:191-203.

Tanksley, S.D., and S.R. McCouch. 1997. Seed banks and molecular maps: Unlocking genetic potential from the wild. Science 277:1063-1066.

Till, B.J., S.H. Reynolds, C. Weil, N. Springer, C. Burtner, K. Young, E. Bowers, C.A. Codomo, L.C. Enns, A.R. Odden, E.A. Greene, L. Comai, and S. Henikoff. 2004. Discovery of induced point mutations in maize genes by TILLING. BMC Plant Biol. 4:12, doi:10.1186/1471-2229-4-12.

Tracy, W.F. 1997. History, genetics and breeding of super sweet (shrunken2) sweet corn. Plant Breed. Rev. 14:189-236.

Whitt, S.R., and E.S. Buckler. 2003. Using natural allelic diversity to evaluate gene function. Methods Mol. Biol. 236:123-140.

Yang, J., W. Wu, and J. Zhu. 2008. Mapping interspecific genetic architecture in a host-parasite interaction system. Genetics 178:1737-1743.

Yu, J., J.B. Holland, M.D. McMullen, and E.S. Buckler. 2008. Genetic design and statistical power of nested association mapping in maize. Genetics 178:539-551.

Zamir, D. 2001. Improving plant breeding with exotic genetic libraries. Nat. Rev. Genet. 2:983-989. 